\title{
Starspots, cycles, and magnetic fields
}

\author{
Steven H. Saar ${ }^{1}$ \\ ${ }^{1}$ Smithsonian Astrophysical Obs., \\ 60 Garden Street, Cambridge, MA 02138, USA \\ email: saar@cfa.harvard.edu
}

\begin{abstract}
I make a perhaps slightly foolhardy attempt to synthesize a semi-coherent scenario relating cycle characteristics, starspots, and the underlying magnetic fields with stellar properties such as mass and rotation. Key to this attempt is to first study single dwarfs; differential rotation plays a surprising role.
\end{abstract}

Keywords. Stars: magnetic fields, stars: spots, stars: rotation, stars: coronae, stars: activity, stars: late-type, stars: evolution

\section{Introduction}

Starspots are one of the most visible manifestations of stellar magnetism, and their periodic variation is often taken evidence for magnetic cycles. The most successful models of cyclic dynamos involve the interaction of differential rotation (DR) and helical turbulence to effect a polarity reversal and drive the cycle (in mean-field terms, $\alpha \Omega$ or $\alpha^{2} \Omega$ dynamos). However, a recent broad-based survey of surface DR measurements (the angular shear $\Delta \Omega$ ) in cool stars Barnes et al. (2005) showed virtually no dependence on rotation: $\Delta \Omega \propto \Omega^{0.15}$. Fundamentally, it is difficult to explain the clear, strong increase in magnetic activity with rotation (by a factor of $\sim 10^{4}$ in coronal emission, for example) if DR has virtually no $\Omega$ dependence (it seems unlikely that the $\alpha$ effect can have such a strong $\Omega$ dependence to compensate; Durney et al. (1993). Thus we are left with the profoundly puzzling situation of a magnetic dynamo whose products show strong rotational dependence, while its component parts (DR, $\alpha$ effect) show little such dependence.

I would like to propose a different way of looking at the data which is physically motivated, and in the end recovers an important, and in some aspects, surprising role for DR in magnetic field generation and cycles. The basic idea is an outgrowth of work presented last year Saar (2009), extended by additional data. I then see how this new view of DR may help to better understand the behavior of cycle periods, amplitudes, and starspot amplitudes and distributions (see also Saar et al. 2010, these proceedings).

\section{DR Data and Analysis}

The Barnes et al. (2005) DR sample is quite heterogeneous, including evolved stars, binaries, and a wide range of spectral types. Saar (2009) proposed that the sample was too broad, mixing categories (e.g., evolved and main sequence, binaries and single) which would more safely be analyzed separately, to better isolate the various parameters which may affect DR. I continue this line of thinking here, updating the Saar (2009) sample with more recent results e.g., (Morin et al. 2008; Lanza et al. 2009; Katsova et al. 2010). The sample is composed of single (or wide binary) dwarfs, later than F5 (B-V>0.46). The latter restriction avoids concerns that hotter stars may have quite different magnetic 
generation processes (possibly due to the extreme thinness of their convection zones) as suggested by lack of any rotation-activity relationship Böhm-Vitense et al. (2002). Where multiple measurement exist (some stars may have variable DR; Jeffers et al. (2007)), I use the highest $\mathrm{DR}$ value as indicative of the maximum strength of the dynamo for a given set of stellar conditions. I also require stars with rotation period $\left(P_{\text {rot }}\right)$ measurements, to avoid $\sin i$ uncertainties, and systematics from activity-rotation based estimates (unfortunately, this rules out most DR values determined from the Fourier transform method, as most of these stars have only $v \sin i$ data; Reiners \& Schmitt (2003).

Several important caveats should be noted. Although the DR sample is pruned, the data themselves are also heterogeneous, with several quite different detection methods used. Timing methods (measuring drifts in $P_{\text {rot }}$ from photometry or Ca II HK) give lower limits to DR. There is also the general worry that $P_{\text {rot }}$ derived from photometry/HK can be difficult to interpret properly when the star is highly spotted Eaton et al. (1996); Jeffers (2005). Doppler imaging methods can give better DR values, but are subject to extrapolation uncertainties when features are only visible in a limited range of latitudes, and systematics in latitude placement. Fourier methods in principle derive DR over the whole surface, but are confused by spots (especially at the poles).

With these issues in mind, I analyze the expanded Saar (2009) sample for trends. Figure 1 shows a clear relationship $\Delta \Omega \propto \Omega^{0.68}\left(\sigma_{\text {fit }}=0.246 \mathrm{dex}\right)$ for $\Omega \leqslant 3 \mathrm{~d}^{-1}$ $\left(\approx 12 \Omega_{\odot}\right)$, already quite different from the Barnes et al. $(2005)$ result $\left(\propto \Omega^{0.15}\right)$, but very similar to the old HK-based result $\left(\propto \Omega^{0.7}\right.$; (Donahue et al. 1996, Donahue et al. 1996). There is also reasonable agreement with detailed 3-D hydrodynamic models, which find $\Delta \Omega \propto \Omega^{0.4}$ (Brown et al. 2008, Brown et al. 2008). The relationship disappears for $\Omega>3$ $\mathrm{d}^{-1}\left(\approx 12 \Omega_{\odot}\right)$, but a new, color dependent one emerges: $\log _{10} \Omega \propto-2.27(\mathrm{~B}-\mathrm{V})$ (Fig. 1 . right; $\sigma_{\text {fit }}=0.129$ dex; throughout, the smallest $\Delta \Omega$ value is neglected from the fits as an outlier). This suggests DR in rapid rotators may depend on $T_{\text {eff }}$ (cf. (Barnes et al. 2005, Barnes et al. 2005)) or the convective turnover timescale $\tau_{C}$, which also produce reasonable fits.
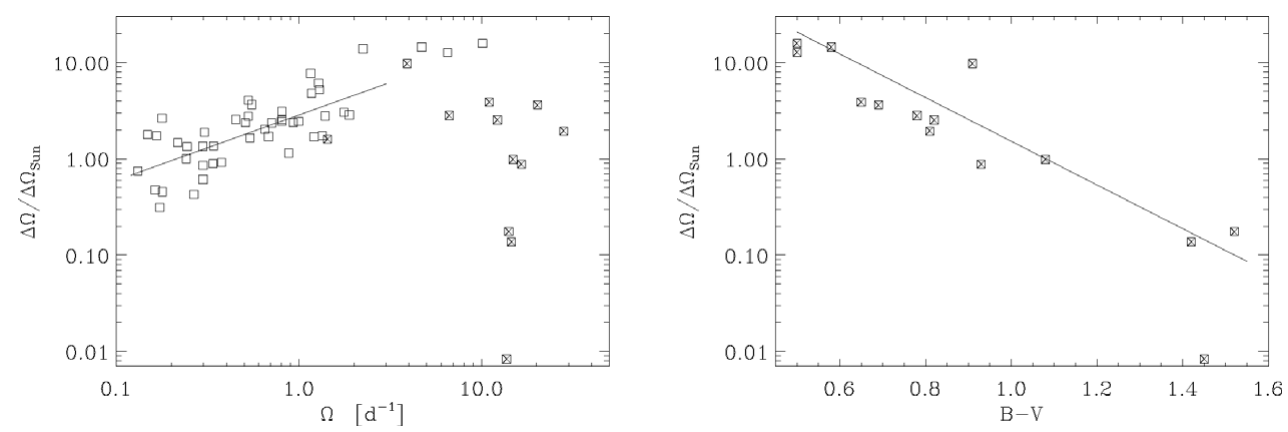

Figure 1. [Left]: Surface DR $\Delta \Omega$ (normalized to the solar value) vs. $\Omega$ for the sample, saturated activity (SA) stars (see Fig. 2, right) are crossed. A least squares fit $\Delta \Omega \propto \Omega^{0.68}$ (solid) is shown for $\Omega<3 \mathrm{~d}^{-1}$. [Right]: Normalized $\Delta \Omega$ vs. B-V color for stars with $\Omega>3 \mathrm{~d}^{-1}$.

The latter fit suggests one physically motivated way to combine the $\Omega$ and color relationships would be through the inverse Rossby number $\mathrm{Ro}^{-1}=\tau_{C} \Omega$ (I take $\tau_{C}$ from Gunn et al. (1998). This idea has the added advantage of tying DR in with the mean-field dynamo number $\left(\propto \mathrm{Ro}^{-2}\right)$ and with rotational evolution (Barnes \& Kim 2010, Barnes \& Kim 2010). When DR is plotted against Ro ${ }^{-1}$ (Fig. 2, left), the 
high rotation portion collapses onto a single relationship, and the low rotation portion is mostly reshuffled, yielding $\Delta \Omega \propto \mathrm{Ro}^{-1.00}\left(\sigma_{\mathrm{fit}}=0.244 \mathrm{dex}\right)$ for $\mathrm{Ro}^{-1}<80$, and $\Delta \Omega \propto \mathrm{Ro}^{1.28}$ for $\mathrm{Ro}^{-1}>80\left(\sigma_{\text {fit }}=0.367 \mathrm{dex}\right)$. Thus, not only does $\Delta \Omega$ in single dwarfs appear to have a significant $\Omega$ dependence, it reverses its strong dependence at high $\mathrm{Ro}^{-1}$.
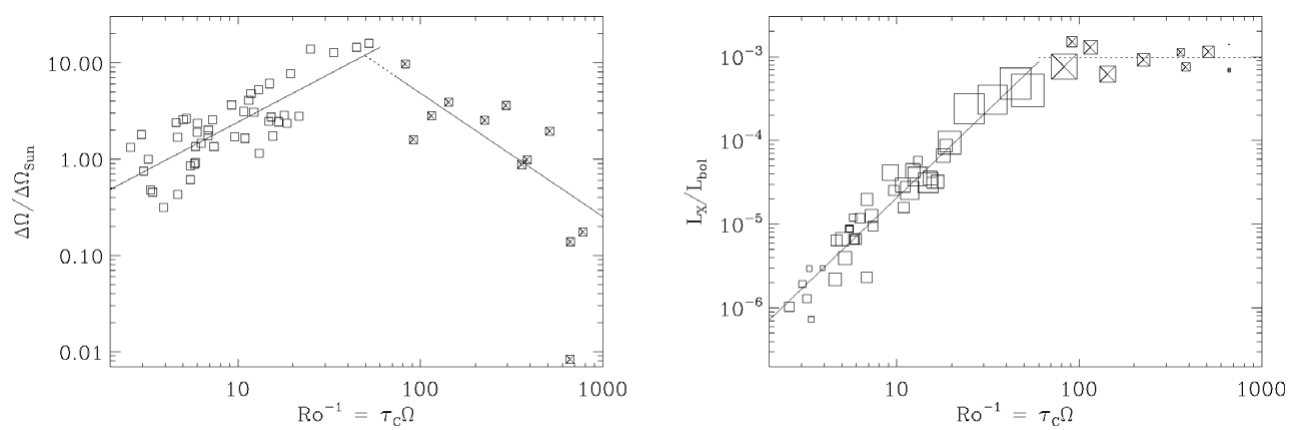

Figure 2. [Left]: Normalized $\Delta \Omega$ vs. $\mathrm{Ro}^{-1}=\tau_{C} \Omega$; symbols as in Fig. 1. Least square fits of $\Delta \Omega$ vs. $\mathrm{Ro}^{-1.00}\left(\mathrm{Ro}^{-1}<80\right)$ and $\Delta \Omega$ vs. $\operatorname{Ro}^{1.28}\left(\mathrm{Ro}^{-1}>80\right)$ are shown (solid). [Right]: $L_{X} / L_{\text {bol vs. }}$. $\mathrm{Ro}^{-1}$; symbols as in Fig. 1 , with size scaling as $(\Delta \Omega)^{0.5}$. A fit with $L_{X} / L_{\mathrm{bol}} \propto \mathrm{Ro}^{-2.088}$ (solid) together with the SA level (dotted) is indicated.

The striking trend reversal in $\Delta \Omega$ finds an impressive parallel in the traditional rotation-activity relationship. It has long been known that various measures of magnetic activity reach a maximum level - "saturate" - above some critical rotation level. If one studies the fractional (relative to bolometric) X-ray luminosity $L_{X} / L_{\mathrm{bol}} \mathrm{vs} \mathrm{Ro}^{-1}$ for the DR sample (X-ray data from ROSAT All Sky Survey; calibration from Hünsch et al. (1999), it is immediately apparent that saturated activity (=SA) occurs at the peak in DR: at $\mathrm{Ro}^{-1} \approx 80$ (Fig. 2, right). This can be even more strikingly demonstrated by directly plotting $L_{X} / L_{\text {bol }}$ against DR (Fig. 3, left). The diagram splits into two radically different regimes, giving the appearance of a large number "7": a low activity regime showing $L_{X} / L_{\mathrm{bol}} \propto \Delta \Omega^{1.36}\left(\sigma_{\mathrm{fit}}=0.428 \mathrm{dex}\right)$ and a SA regime where coronal emission is independent of $\Delta \Omega$ over 3 orders of magnitude in rotational shear. Since $L_{X}$ is proportional to magnetic flux over many decades (Pevtsov et al. (2003)), the implication is both clear and surprising: in the SA regime, a maximal amount of coronal/magnetic flux can be generated by stellar dynamos independent of the differential rotation rate. This would appear to be striking proof that a fundamentally different kind of dynamo dominates the $S A$ realm. Hints of this have already arisen in the persistence of low latitude magnetic features on rapid rotators (e.g., Deluca et al. (1997)).

It is also informative to explore the time dependence of DR. I use the latest formulation of gyrochronology, which uses $\Omega$ and $\tau_{C}$ to derive stellar ages, $t$ (Barnes \& Kim 2010, Barnes \& Kim 2010). When DR is plotted versus gyrochronological age, a gently decline of DR with time is seen (Fig. 3, right). When SA stars $\left(\mathrm{Ro}^{-1}>80\right)$ are excluded, the relationship tightens considerably, yielding $\Delta \Omega \propto t^{-0.57}\left(\sigma_{\mathrm{fit}}=0.232 \mathrm{dex}\right)$. The $\Delta \Omega$ in SA stars apparently increases, evolving up to this line. There is also a color/mass dependent effect: high mass stars appear on the declining sequence first, with lower mass stars joining later. The paucity of stars limits accuracy, but F8 stars appear to join the decline by $t \sim 30 \mathrm{Myr}$, G1 by $\sim 70 \mathrm{Myr}, \mathrm{K} 2$ by $\sim 200 \mathrm{Myr}, \sim 500 \mathrm{Myr}$ for K5, and $\sim 2$ Gyr at M4. This roughly corresponds to when stars of these masses leave the SA regime. The 

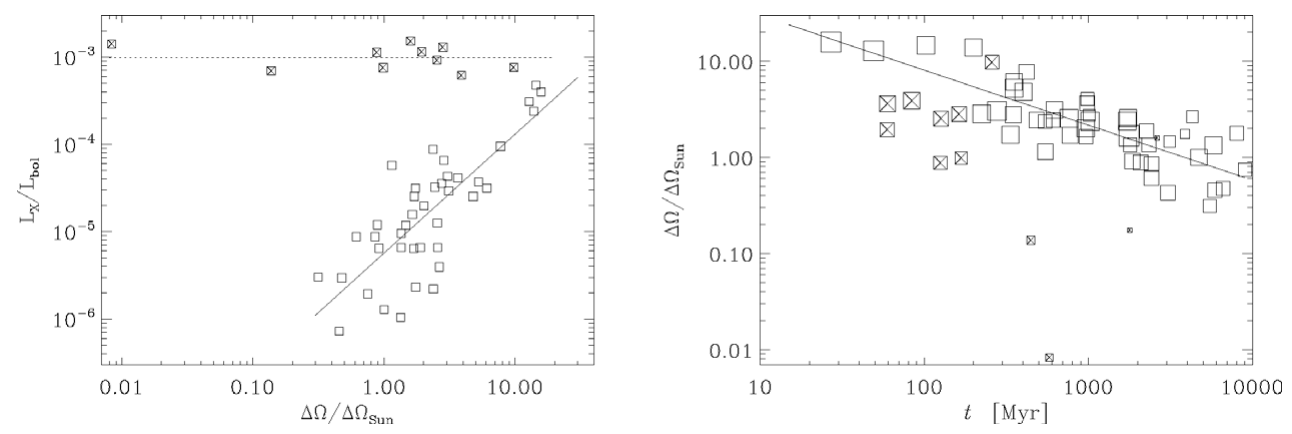

Figure 3. [Left]: $L_{X} / L_{\mathrm{bol}}$ vs. normalized $\Delta \Omega$, showing the fit $L_{X} / L_{\mathrm{bol}} \propto \Delta \Omega^{1.36}$ (solid) and SA level (dotted); symbols as in Fig. 1. [Right]: Normalized $\Delta \Omega$ vs. age $t$ (from (Barnes \& Kim 2010, Barnes \& Kim 2010)). Symbols as in Fig. 1, with size scaling with stellar mass (1.15 to $0.3 M_{\odot}$; from (Barnes \& Kim 2010, Barnes \& Kim 2010)). A power law fit to the non-SA stars $\Delta \Omega \propto t^{-0.57}$ (solid) is also shown.

notable similarity of the power law with the classic Skumanich (1972) $v \propto t^{-0.5}$ empirical spindown law, together with the mass dependent start of the spindown, strongly hint that $D R$ has an important role in controlling the rotational evolution of cool stars. We will explore this further in an upcoming paper (Barnes \& Saar 2010, in prep.)

\section{Cycle Data and Analysis}

Armed with this new understanding of the $\Omega$ and mass dependence of DR in single dwarfs, I now turn to the question of cycle properties. I adopt identical selection criteria as for the DR sample, and also insist vis. Saar \& Brandenburg (1999) that the cycle be a relatively "clean", high quality detection (this is admittedly subjective in some cases). We adopt the cycle sample of Saar (2009), with some recent additions (e.g., (Oláh et al. 2009; Fares et al. 2009); Milingo et al. 2010, these proceedings). A quick look at the data reveals no strong trend with $\Omega$ (as found by, e.g., (Oláh et al. 2009). However, if one separates the secondary (= weaker amplitude) cycle period $P_{\text {cyc }}(2)$, present in many moderate-to-active stars, and insists that they somehow be in a relationship separate from the main stellar cycle e.g, (Soon et al. 1993; Saar \& Brandenburg 1999), a more complex, multitiered pattern emerges (Fig. 4). When cycle frequency $\omega_{\text {cyc }}$ is plotted against $\Omega$, three parallel tracks, separated by a factor of $\sim 3.5$, each with $\omega_{\text {cyc }} \propto \Omega^{1.1}$. At $\Omega \approx 10 \Omega \odot$, very close to DR peak (Fig. 1, right), the relations reverse, showing $\omega_{\text {cyc }} \propto \Omega^{-0.6}$ (Fig. 4). Only six of the 15 double cycle stars fail to have their $P_{\text {cyc }}$ on separate branches. Thus the DR peak would appear to have left a mark in $\omega_{\text {cyc }}$ as well.

Key to this interpretation of the cycle data is the reality of the $P_{\text {cyc }}(2)$ as true, separate, polarity-reversing cycles (rather than just amplitude modulations of the main cycle). While this is not known to be true in general, there is evidence supporting this idea. First, $P_{\text {cyc }}(2)$ are generally short, and recent Zeeman Doppler imaging evidence demonstrates that at least some short cycles do reverse polarity (Fares et al. 2009; Petit et al. 2009, Fares et al. 2009; Petit et al. 2009). Second, the amplitudes of the primary and secondary cycles show opposite trends with increasing $\mathrm{Ro}^{-1}$ Moss et al. (2008), suggesting they have different physical sources Böhm-Vitense (2007); possibly related to higher order dynamo modes Petit et al. (2009). The amplitudes of many cycles vary in time though Oláh et al. (2009), so assigning a single amplitude to each cycle is likely too simplistic. 


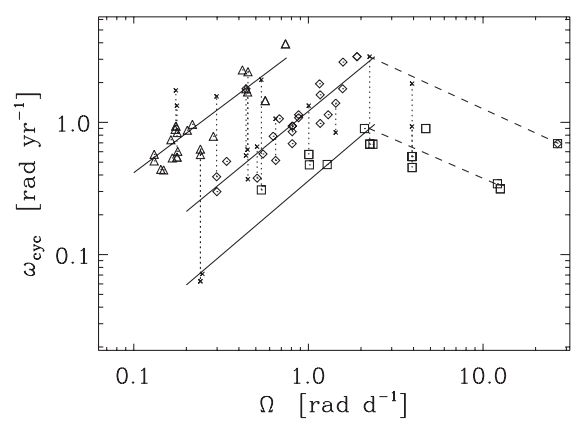

Figure 4. Cycle frequency $\omega_{\text {cyc }}$ versus $\Omega$ for the dataset, triangles, diamonds, and squares represent stars assigned to the three "branches". Power law fits yield $\omega_{\text {cyc }} \propto \Omega^{1.1}$ and $\sigma_{\text {fit }} \approx$ 0.12 dex for each branch below $10 \Omega_{\odot}$ (at DR peak and the start of mass-dependent drop in $\Delta \Omega$; see Fig. 1 left). The (less well defined) descending portions at high $\Omega$ are consistent with $\omega_{\text {cyc }} \propto \Omega^{-0.6}$. A single fit to all the data yields $\omega_{\text {cyc }} \propto \Omega^{0.15}$ with considerably higher scatter $\left(\sigma_{\text {fit }}=0.33\right.$ dex; not shown $)$.

\section{Other Implications and Connections}

The DR results for the sample adopted here show a strong, two regime $\Omega$ dependence. Yet Barnes et al. (2005), with a similar sample which, however, also included binaries and evolved stars, found much weaker $\Omega$ dependence, and lower overall average DR values (compare their Fig. 3 with Fig. 1 here). This suggests that binary and/or evolved stars must have weaker DR, which likely also has weaker $\Omega$ dependence than the present (dwarf, single) sample. A detailed investigation of this is beyond the scope of this paper, but is clearly worthwhile. I note that there has already been hints of a binary/single difference in how $\Omega$ evolves in clusters. If binaries do have lower DR, and DR is related to spindown (e.g., Fig. 3, right), this may explain the slower than expected rotation rates seen in some cluster binaries Meibom et al. (2006).

The sharp peak in DR may also have an effect on starspot distributions. There is a sharp drop in the maximum observed photometric spot amplitudes $A_{\max }$ at nearly the same $\mathrm{Ro}^{-1}(\approx 100)$ as the DR peak (Saar et al. 2010, these proceedings). The $\sigma$ width, skewness, and kurtosis of the spot amplitude distributions also drop sharply near the DR peak. The authors speculate that strong DR may strongly shear spot groups, increasing homogeneity, and thereby decreasing $A_{\max }$ and distribution moments.

Spectropolarimetric data has recently been starting to reveal large scale topologies in cool stars (Morin 2010, these proceedings). The DR peak corresponds roughly where dynamos transition to a more axisymmetric, poloidal geometry. An earlier transition from solar-like poloidal to strongly toroidal, non-axisymmetric geometry Petit et al. (2008) may, combined with the sharp $A_{\text {max }}$ peak around $\mathrm{Ro}^{-1} \sim 60$ (Saar et al. 2010, these proceedings), may be the observational signature of the formation of powerful convectionzone dynamos and toroidal magnetic "wreathes" (Brown et al. 2010, Brown et al. 2010). Tachocline-driven dynamos may only be prominent at relatively low $\mathrm{Ro}^{-1}$, since meridional flows needed to sustain them drop with $\Omega$ (Brown et al. 2008; Jouve et al. 2010).

\section{Summary and Conclusions}

To summarize the results: in single dwarfs, (1) DR has a strong, two part dependence on rotation, increasing as $\Delta \Omega \propto \mathrm{Ro}^{-1}$ for coronally un-saturated stars and as $\mathrm{Ro}^{1.38}$ 
for saturated activity stars. (2) DR peaks just at the low $\mathrm{Ro}^{-1}$ end of the SA branch. (3) The lack of correlation between $L_{X} / L_{\mathrm{bol}}$ and $\Delta \Omega$ on the SA branch implies the dynamo mechanism is quite different there than in un-saturated stars. (4) The age/mass dependence of DR (nearly identical to that for $\Omega(t)$ ) suggest DR plays an important role in rotational evolution. (5) The peak in DR has an echo in the multitiered pattern of $\omega_{\text {cyc }}$ values, which also change power law dependence roughly at the $\Omega$ and Ro that DR does. (6) The peak in DR is also the location of a local minimum in the maximum spot amplitudes; peak DR may shear spot groups, preventing larger $A_{\text {max }}$ values. The moments of the $A_{\text {spot }}$ distributions also change sharply near DR peak. (7) Comparing these results with Barnes et al. (2005) suggests that binaries likely have reduced DR, possibly with weaker dependence on $\Omega$ and $\mathrm{Ro}^{-1}$. (8) Peak DR may correspond to a transition in dynamo symmetry from toroidal and non-axisymmetric (at lower $\mathrm{Ro}^{-1}$ ) towards more poloidal and axisymmetric (at higher $\mathrm{Ro}^{-1}$ ).

There is a clear need to study DR in binaries, and expand investigations to the preand post-MS. But it is also important to realize that the present results may well be revised, or even overturned, very quickly! COROT and Kepler will be soon be flooding us with vast mountains of new $A_{\text {spot }}, P_{\text {rot }}$, and $\Delta \Omega$ measurements. All these will surely help us better understand the complex workings of stellar dynamos and their myriad products... or at least provide much stimulating confusion!

Acknowledgments: This work was supported by a Solar Heliospheric Guest Investigator grant NNX10AF29G, and by Chandra grants GO8-9025A and GO0-11041A. I am indebted to S. Barnes, B. Brown, M. Browning, S. Meibom, A. Muñoz-Jaramillo, D. Nandy, K. Olah, and M. Rempel, among many others, for helpful conversations.

\section{References}

Böhm-Vitense, E. 2007, Astrophys. J., 657, 486

Böhm-Vitense, E., Robinson, R., Carpenter, K., \& Mena-Werth, J. 2002, Astrophys. J., 569, 941

Barnes, J. R., Cameron, A. C., Donati, J.-F., James, D. J., Marsden, S. C., \& Petit, P. 2005, Mon. Not. Roy. Astron. Soc., 357, L1

Barnes, S. A. \& Kim, Y.-C. 2010, Astrophys. J., 721, 675

Brown, B. P., Browning, M. K., Brun, A. S., Miesch, M. S., \& Toomre, J. 2010, Astrophys. J., 711,424

Brown, B. P., Browning, M. K., Brun, A. S., Miesch, M. S., \& Toomre, J. 2008, Astrophys. J., 689,1354

Deluca, E. E., Fan, Y., \& Saar, S. H. 1997, Astrophys. J., 481, 369

Donahue, R. A., Saar, S. H., \& Baliunas, S. L. 1996, Astrophys. J., 466, 384

Durney, B. R., De Young, D. S., \& Roxburgh, I. W. 1993, Solar Phys., 145, 207

Eaton, J. A., Henry, G. W., \& Fekel, F. C. 1996, Astrophys. J., 462, 888

Fares, R., et al. 2009, Mon. Not. Roy. Astron. Soc., 398, 1383

Gunn, A. G., Mitrou, C. K., \& Doyle, J. G. 1998, Mon. Not. Roy. Astron. Soc., 296, 150

Hünsch, M., Schmitt, J. H. M. M., Sterzik, M. F., \& Voges, W. 1999, A\&SAS, 135, 319

Jeffers, S. V. 2005, Mon. Not. Roy. Astron. Soc., 359, 729

Jeffers, S. V., Donati, J.-F., \& Collier Cameron, A. 2007, Mon. Not. Roy. Astron. Soc., 375, 567

Jouve, L., Brown, B. P., \& Brun, A. S. 2010, Astron. Astrophys, 509, A32

Katsova, M. M., Livshits, M. A., Soon, W., Baliunas, S. L., \& Sokoloff, D. 2010, NewA, 15, 274

Lanza, A. F., et al. 2009, Astron. Astrophys, 506, 255

Meibom, S., Mathieu, R. D., \& Stassun, K. G. 2006, Astrophys. J., 653, 621

Morin, J., et al. 2008, Mon. Not. Roy. Astron. Soc., 384, 77

Moss, D., Saar, S. H., \& Sokoloff, D. 2008, Mon. Not. Roy. Astron. Soc., 388, 416

Oláh, K., et al. 2009, Astron. Astrophys, 501, 703 
Petit, P., Dintrans, B., Morgenthaler, A., van Grootel, V., Morin, J., Lanoux, J., Aurière, M., \& Konstantinova-Antova, R. 2009, Astron. Astrophys, 508, L9

Petit, P., et al. 2008, Mon. Not. Roy. Astron. Soc., 388, 80

Pevtsov, A. A., Fisher, G. H., Acton, L. W., Longcope, D. W., Johns-Krull, C. M., Kankelborg, C. C., \& Metcalf, T. R. 2003, Astron. Astrophys, 598, 1387

Reiners, A. \& Schmitt, J. H. M. M. 2003, Astron. Astrophys, 398, 647

Saar, S. H. 2009, Stellar Dynamos as Revealed by Helio- and Asteroseismology, ASP Conf. Ser. 416,375

Saar, S. H. \& Brandenburg, A. 1999, Astrophys. J., 524, 295

Skumanich, A. 1972, Astrophys. J., 171, 565

Soon, W. H., Baliunas, S. L., \& Zhang, Q. 1993, Astrophys. J. 1, 414, L33 